\title{
Experimental Investigation of Phase Noise Tolerance of SSB THz Signals
}

\author{
Luis Gonzalez Guerrero*, Haymen Shams, Martyn J. \\ Fice, Alwyn J. Seeds and Cyril C. Renaud \\ Department of Electronic and Electrical Engineering \\ University College London \\ London, UK \\ *luis.guerrero.14@ucl.ac.uk
}

\author{
Irshaad Fatadin, Mira Naftaly \\ Electromagnetic Technologies group \\ National Physical Laboratory \\ London, UK
}

\author{
Frédéric van Dijk \\ III-V Lab, a joint Laboratory of "Alcatel Lucent Bell Labs" \\ "Thales Research \& Technology" and "CEA-LETT" \\ Palaiseau, France
}

\begin{abstract}
Wireless bridges operating at $\mathrm{THz}$ frequencies have drawn a lot of attention in recent years. This application requires multiple optical local oscillators (LOs) throughout the link and, hence, the accumulated phase noise may impose a major challenge for its practical implementation. Of all the techniques to mitigate phase noise, single sideband (SSB) modulation is a very promising candidate due to its simple implementation. In this paper, we show, for the first time, the transmission of a $6 \mathrm{GBd}$ SSB QPSK signal at $250 \mathrm{GHz}$ and investigate the impact of laser phase noise on the quality of the THz signal. The performance of the system is investigated by digitally tuning the linewidth of the optical local oscillator up to $10 \mathrm{MHz}$. The results show no observable penalty associated with increasing linewidth, confirming the validity of SSB for applications where multiple domain conversions are required.
\end{abstract}

Keywords-THz communications, Optical communications, Wireless bridges, Single sideband modulation.

\section{INTRODUCTION}

For 5G mobile systems, data rates of up to $100 \mathrm{Gbit} / \mathrm{s}$ will be required in the backhaul links of both wireless and optical access networks [1]. Although optical fiber is likely to be the preferred option for this type of link due to its large bandwidth, its deployment will not always be economical, as for example in crowded urban areas or difficult-to-access terrains. In this scenario, a high-speed wireless link at $\mathrm{THz}$ frequencies $(100$ $\mathrm{GHz}-10 \mathrm{THz}$ ) can be advantageous. Compared to mm-waves, $\mathrm{THz}$ would enable the use of lower order modulation formats because of its higher bandwidth. On the other hand, unlike free-space optical (FSO) communications, THz links would ensure operability under adverse atmospheric conditions such as fog, or in the presence of airborne particles as in smoke or dust [2]. Among the applications that are envisaged for $\mathrm{THz}$ communications, wireless bridges (wireless links embedded in a fiber link) have drawn considerable attention in recent years (see Fig. 1 for a schematic of a wireless bridge) [3].
Several demonstrations of wireless bridges have been described in literature [4]-[5]. In [4], a $20 \mathrm{Gbit} / \mathrm{s}$ wireless bridge operating at $220 \mathrm{GHz}$ was demonstrated. There, the $\mathrm{THz}$ generation was performed by means of electronic multiplication. This has the disadvantage of requiring an electronic local oscillator (LO) and a $\mathrm{THz}$ harmonic mixer at the transmitter remote antenna unit (Tx RAU), in addition to a photodiode (PD) to downconvert the optical signal to baseband. To reduce the cost of the RAU, a scheme based on optical heterodyning in a fast PD would be advantageous. In [6], a link using optical heterodyning at the Tx RAU and operating at $95 \mathrm{GHz}$ and $128 \mathrm{Gbit} / \mathrm{s}$ was realized. As noted in Fig. 1, in these links at least 4 lasers (plus two for any additional wireless bridge present in the link) are required for the multiple domain conversions that take place. In such cases, the combined linewidth can amount to several $\mathrm{MHz}$, which might prevent the coherent receiver $(\mathrm{Rx})$ from recovering the electrical signal [7]. In [6], this problem was tackled by using 4 external cavity lasers (ECLs) with narrow linewidths $(<100$ $\mathrm{kHz}$ ). These lasers, however, can be significantly bulky and quite expensive, and are best suited for laboratory use exclusively. In [5], a scheme based on an optical frequency comb generator (OFCG) and two fiber lasers with linewidths of $15 \mathrm{~Hz}$ was demonstrated. Again, the use of such lasers instead of tunable integrated lasers (with linewidths of a few $\mathrm{MHz}$ ) would limit the practical implementation of these systems.

An alternative way to minimize the impact of phase noise from optical LOs is by using phase noise robust modulation formats such as single sideband (SSB) together with envelope detection [8]. In this paper, we demonstrate the transmission of a 6 GBd quadrature phase-shift keying (QPSK) SSB signal at $250 \mathrm{GHz}$, and investigate the effect of LO phase noise. This is done by digitally tuning the LO linewidth up to $10 \mathrm{MHz}$. For this demonstration, we use a harmonic mixer for intermediate frequency (IF) down conversion, and then perform envelope detection in the digital domain after analog-to-digital conversion (ADC). In a practical system, envelope detection 
would be implemented in the analog domain so as to remove the need for an electronic LO at the Rx RAU, as noted in Fig.1. The results obtained in this paper in terms of linewidth tolerance, however, should be equally applicable to a full analog implementation.

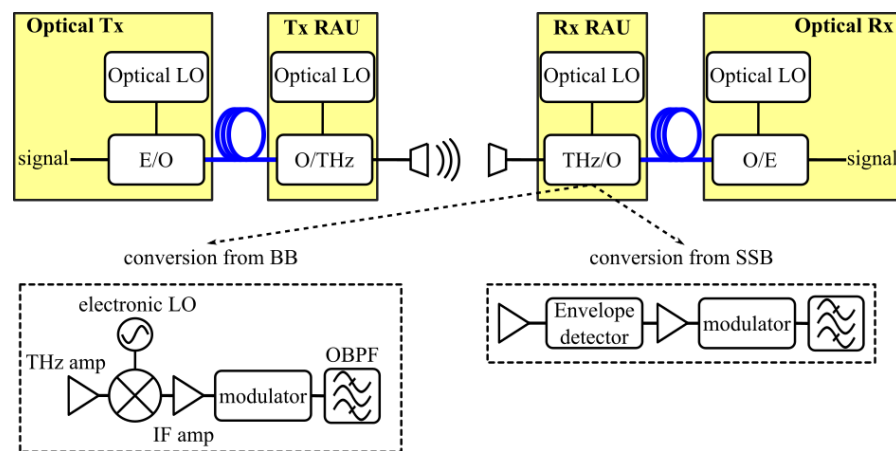

Fig. 1. THz wireless bridge based on optical heterodyning at the Tx remote antenna unit (RAU). A single sideband (SSB) THz signal would be beneficial in terms of phase noise (as we demonstrate in this paper) and would also simplify the Rx RAU design. E/O: electrical-to-optical converter, O/THz: optical-to- $\mathrm{THz}$ converter, $\mathrm{THz} / \mathrm{O}$ : THz-to-optical converter, O/E: optical-toelectrical converter, BB: baseband, OBFF: optical band pass filter.

\section{EXPERIMENTAL PROCEDURE}

\section{A. Generation of the optical SSB signal}

First, a root raised cosine (RRC) shaped QPSK signal (rolloff factor of 0.3 ) was generated in Matlab. Then, the signal was digitally up-converted to an IF. Finally, to remove the lower sideband, a Hilbert transform (HT) was applied to the resultant waveform [9]. In this case the up-conversion frequency was equal to the symbol rate (single-cycle subcarrier modulation SCSM), which was $6 \mathrm{GBd}$ (for a total bit rate of $12 \mathrm{Gbit} / \mathrm{s}$ ). This up-conversion frequency determines the width of the guard band between sideband and carrier. Hence, to maximize the spectral efficiency of the signal, it should be minimized. There are digital signal processing (DSP) algorithms that mitigate the signal-signal beat interference (SSBI) to enable the use of narrow guard bands [10]. If the application allows for a digital $\mathrm{Rx}$ to be implemented after envelope detection, then these DSP algorithms may be a feasible solution. In the scenario that we envisage in this paper (Fig. 1), however, a full analog receiver at the Rx RAU would be advantageous for achieving low latency. In such case, it is necessary to find a compromise between signal quality and spectral efficiency. Given the large bandwidth found in the first sub-THz window $(\sim 73 \mathrm{GHz}, 252 \mathrm{GHz}-325 \mathrm{GHz})$, SCSM might be a reasonable choice.

The maximum suppression ratio that can be achieved for SSB signals generated through the HT is approximately equal to the extinction ratio (ER) of the optical modulator (in our case, an in-phase and quadrature, "IQ", modulator) [11]. However, this upper limit can only be achieved if the I and Q signals have matched time delays and amplitudes when they reach the optical modulator. To ensure identical time delay in both electrical arms, two phase shifters were placed after the arbitrary waveform generator (AWG). For amplitude matching, the electrical signals were pre-equalized. The amplitude responses of the two paths were measured by sending a frequency comb through each arm and interpolating the received signal [12]. This way, in addition to matching the amplitudes of both signals, the high frequency roll-off of the AWG was corrected. The obtained SSB signal is shown in Fig. 2. As can be seen, the suppression ratio is higher than $20 \mathrm{~dB}$ Since the lowest ER of the three Mach-Zehnder modulators (MZM) forming the IQ modulator was $21.5 \mathrm{~dB}$, this confirms that the electrical signals were both time and amplitude matched.

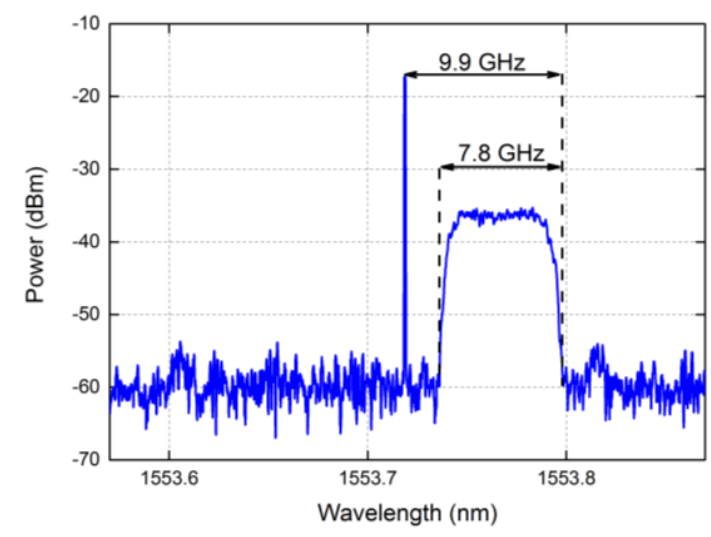

Fig. 2. Optical spectrum of 6 GBd QPSK signal generated after preequalization of the I and Q channels. Resolution BW of $0.1 \mathrm{GHz}$.

\section{B. Transmission setup}

In Fig. 3, the complete experimental setup used for the transmission experiments is shown. On the Tx side, 2 ECLs were used when no emulation of the optical LO was performed. The carrier laser (ECL \#1) has a linewidth of 10 $\mathrm{kHz}$, whereas the ECL used as a LO (ECL \#2) has a linewidth of $100 \mathrm{kHz}$. The two optical tones, set at a frequency difference of $250 \mathrm{GHz}$, were sent to a uni-travelling carrier (UTC) PD after optical amplification and filtering. Fig. 3 inset (a) shows the combined optical signals before the UTC-PD. In this proofof-concept experiment, the signal was transmitted through a very short wireless distance $(<1 \mathrm{~cm})$ to maximize the power budget. Nevertheless, it is expected that $\mathrm{THz}$ links will be able to reach distances of up to a few kilometers as higher power output $\mathrm{THz}$ amplifiers become commercially available.

The received signal was down-converted to an IF of around $11 \mathrm{GHz}$ by mixing it with the 6th harmonic of the output of an RF synthesizer in a sub-harmonic mixer (SHM). The downconverted signal was then sampled by an $80 \mathrm{GSa} / \mathrm{s}$ oscilloscope. After ADC, envelope detection was performed offline. Finally, a typical coherent DSP routine consisting of IQ down-conversion, matched filtering, resampling, normalization and equalization was carried out [13]. The equalizer was initialized using the constant modulus algorithm (CMA). Then, the Viterbi-Viterbi algorithm and a block averaging filter of 100 samples in length was applied for phase correction before switching to a decision-directed (DD) mode equalizer. Finally, symbol decision and bit error counting were performed after differential and grey decoding. 


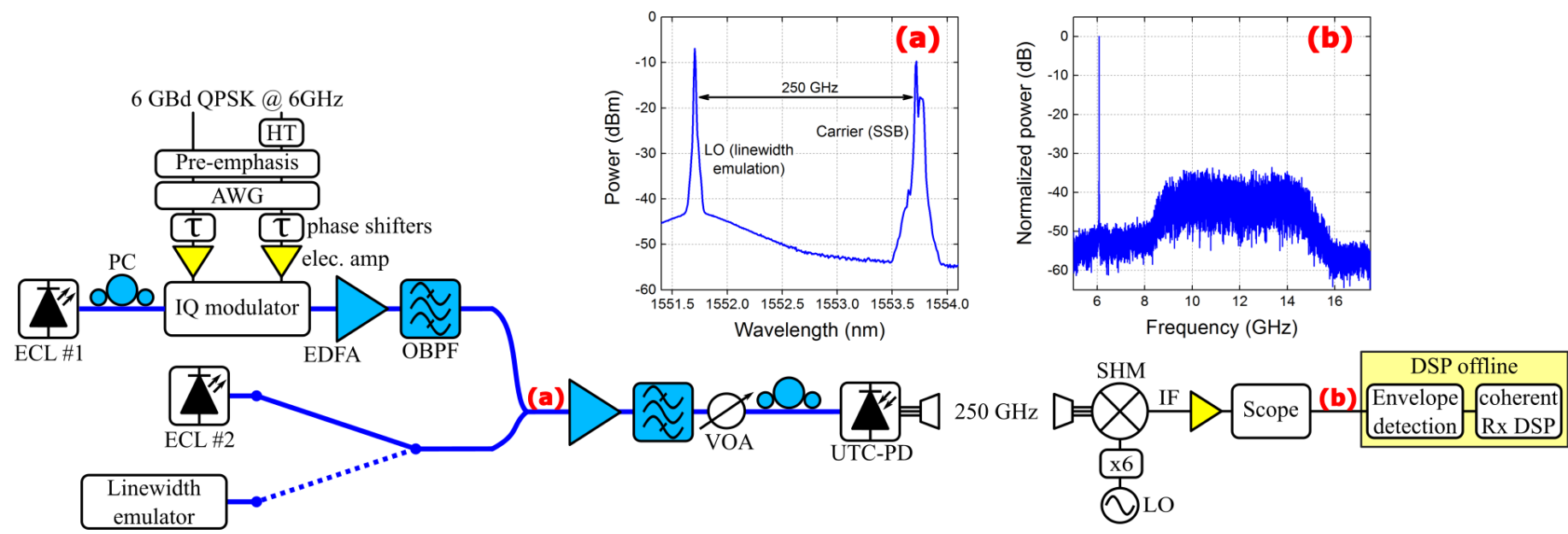

Fig. 3. Experimental setup used for the SSB THz wireless transmission and linewidth emulation. The optical spectrum (Resolution BW of 0.01 nm) before optical mixing is shown in inset (a), while the received signal after ADC is shown in inset (b). VOA: variable optical attenautor, PC: polarization controller, SHM: sub-harmonic mixer.

\section{LO linewidth tuning}

For linewidth tuning, we generated several white frequency noise sequences and modulated them on to ECL \#2 using a second IQ modulator [14]. The linewidth of the THz signal was estimated by measuring the frequency modulation (FM) noise spectrum of the heterodyne signal between ECL \#1 and the output of the linewidth emulator at a frequency of around 3.2 $\mathrm{GHz}$. The beat note at this frequency was recorded by a 20 $\mathrm{GSa} / \mathrm{s}$ oscilloscope and processed digitally offline (down converted, filtered and resampled) before computing the white frequency noise component [15]. The measured Lorentzian linewidths were $580 \mathrm{kHz}, 1.06 \mathrm{MHz}, 5.04 \mathrm{MHz}$ and 10.20 $\mathrm{MHz}$ for emulated linewidths of $500 \mathrm{kHz}, 1 \mathrm{MHz}, 5 \mathrm{MHz}$ and $10 \mathrm{MHz}$ respectively.

\section{RESULTS}

In Fig. 4 (a), the BER curve without linewidth emulation (i.e. combined linewidth of $110 \mathrm{kHz}$ ) is shown as a function of the square of the average photocurrent generated in the UTC$\mathrm{PD}$ (parameter proportional to the generated $\mathrm{THz}$ power). As can be seen, a BER of less than $1.3 \cdot 10^{-3}$ was obtained, confirming that error free transmission can be achieved with the proposed scheme by using hard decision forward error correction (HD-FEC) with 7\% overhead. The inset of Fig. 4 (b) shows the constellation diagram for the lowest BER value, which showed an error vector magnitude (EVM) of $28 \%$. In Fig. 4 (b), the BER curves for all values of measured linewidths (including the no emulation curve) are plotted vs. the square of the photocurrent. As can be seen, no penalty associated with increasing beat note linewidth is observed.

\section{CONCLUSIONS}

$\mathrm{THz}$ communications are a candidate for future high-speed wireless communications. Amongst envisaged applications, wireless bridges impose a challenge in terms of phase noise due to the multiple domain conversions that take place throughout the link. In this paper, we propose SSB as a potential modulation format for this application. We have successfully achieved the transmission of a 6 GBd QPSK SSB signal at $250 \mathrm{GHz}$. We have further investigated the impact of optical LO linewidth on the quality of the received signal. No observable penalty was seen for a linewidth of up to $10 \mathrm{MHz}$, confirming the robustness of this technique in the presence of laser phase noise. This technique therefore allows the use of inexpensive laser sources, such as diode lasers, for this type of application.
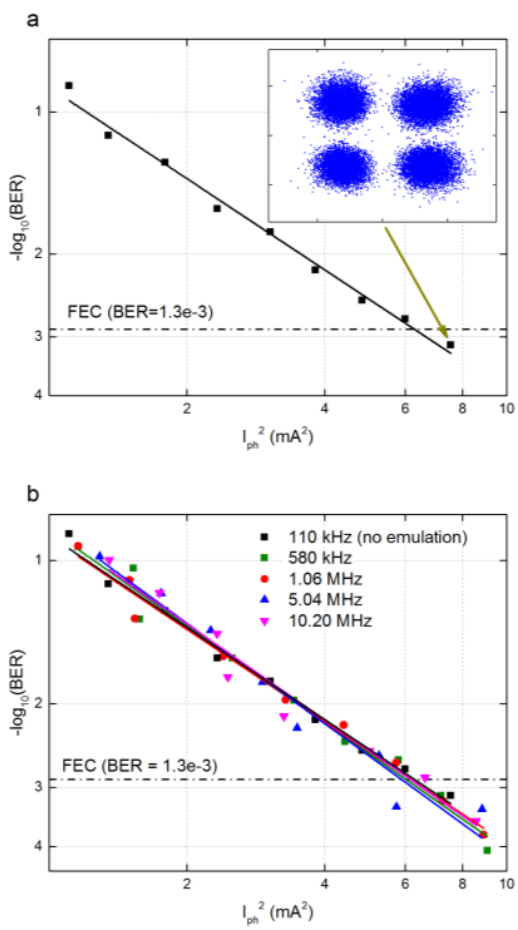

Fig. 4. BER curves as a function of the square of the photocurrent $\left(\mathrm{I}_{\mathrm{ph}}{ }^{2}\right)$ for the case without linewidth emulation (a) and for all the combined linewidths after linewidth emulation (the no emulation curve is also shown for comparison) (b). 


\section{ACKNOWLEDGMENTS}

This work was supported by the Engineering and Physical Sciences Research Council programme grant Coherent Terahertz Systems (COTS) (EP/J017671/1), and by the European Commission through the European project iPHOS (grant agreement no: 257539). The work was also funded by the project EMPIR 14IND13 PhotInd at NPL. This project has received funding from the EMPIR programme co-financed by the Participating States and from the European Union's Horizon 2020 research and innovation programme.

\section{REFERENCES}

[1] S. E. Alavi, M. R. K. Soltanian, I. S. Amiri, M. Khalily, A. S. M. Supa'at, and H. Ahmad, "Towards 5G: A Photonic Based Millimeter Wave Signal Generation for Applying in 5G Access Fronthaul," Sci. Rep., vol. 6, 19891, pp. 1-11, 2016.

[2] A. J. Seeds, H. Shams, M. J. Fice, and C. C. Renaud, "TeraHertz photonics for wireless communications," J. Light. Technol., vol. 33, no. 3, pp. 579-587, 2015.

[3] A. Kanno, P. Tien Dat, T. Kuri, I. Hosako, T. Kawanishi, Y. Yoshida, and K. Kitayama, "Waveform over fiber: DSP-aided coherent fiberwireless transmission using millimeter and terahertz waves," Broadband Access Commun. Technol. IX, vol. 9387, pp. 1-11, 2015.

[4] S. Koenig, J. Antes, D. Lopez-Diaz, I. Kallfass, T. Zwick, C. Koos, W. Freude, and J. Leuthold, "20 Gbit/s Wireless Bridge at $220 \mathrm{GHz}$ Connecting Two Fiber-Optic Links," J. Opt. Commun. Netw., vol. 6, no. 1, pp. 977-981, 2014.

[5] A. Kanno, P. T. Dat, N. Sekine, I. Hosako, N. Yamamoto, Y. Yoshida, K. Kitayama, and L. Fellow, "Seamless Fiber-Wireless Bridge in the Millimeter- and Terahertz-Wave Bands," vol. 34, no. 20, pp. 47944801, 2016

[6] X. Li, J. Yu, J. Xiao, and Y. Xu, "Fiber-wireless-fiber link for 128-Gb/s PDM-16QAM signal transmission at W-band," IEEE Photonics Technol. Lett., vol. 26, no. 19, pp. 1948-1951, 2014.
[7] H. Shams, L. Gonzalez-Guerrero, M. Fice, Z. Yang, C. Renaud, and A. Seeds, "Distribution of multiband $\mathrm{THz}$ wireless signals over fiber," Proc. SPIE 10128, Broadband Access Communication Technologies XI, 101280G, 2017.

[8] K. Bałakier, M. P. Thakur, F. Van Dijk, M. Lamponi, M. Chtioui, Y. Leiba, J. E. Mitchell, A. J. Seeds, and C. C. Renaud, "Demonstration of Photonic Integrated RAU for Millimetre-wave Gigabit Wireless Transmission," in Microwave photonics (MWP), 2016, vol. 6, pp. 344347.

[9] M. S. Erkilinç, S. Kilmurray, S. Pachnicke, H. Griesser, B. C. Thomsen, P. Bayvel, and R. I. Killey, "Nyquist-shaped dispersion-precompensated subcarrier modulation with direct detection," Opt. Express, vol. 22, no. 8, pp. 9420-9431, 2014.

[10] Z. Li, M. S. Erkilinç, S. Pachnicke, H. Griesser, R. Bouziane, B. C. Thomsen, P. Bayvel, and R. I. Killey, "Signal-signal beat interference cancellation in spectrally-efficient WDM direct-detection Nyquist-pulseshaped 16-QAM subcarrier modulation," Opt. Express, vol. 23, no. 8, pp. 23694- 23709, 2014.

[11] M. S. Erkilinc, M. P. Thakur, S. Pachnicke, H. Griesser, J. Mitchell, B. C. Thomsen, P. Bayvel, and R. I. Killey, "Spectrally efficient WDM nyquist pulse-shaped subcarrier modulation using a dual-drive MachZehnder modulator and direct detection," J. Light. Technol., vol. 34, no. 4, pp. 1158-1165, 2016.

[12] F. C. A. Brecht, R. B. Onjour, S. W. Elschen, A. J. Osten, B. B. Aeuerle, D. H. Illerkuss, M. B. Urla, and J. L. Euthold, "Pre-equalization technique enabling $70 \mathrm{Gbit} / \mathrm{s}$ photonic-wireless link at $60 \mathrm{GHz}$," Opt. Express, vol. 24, no. 26, pp. 30350-30359, 2016.

[13] H. Shams, M. J. Fice, L. Gonzalez-Guerrero, C. C. Renaud, F. van Dijk, and A. J. Seeds, "Sub-THz Wireless over Fibre for Frequency Band 220 GHz- 280 GHz," J. Light. Technol., vol. 34, no. 20, pp. 4786-4793, 2016.

[14] Z. Zan and A. J. Lowery, "Experimental demonstration of a flexible and stable semiconductor laser linewidth emulator.," Opt. Express, vol. 18, no. 13 , pp. 13880-13885, 2010.

[15] I. Fatadin, D. Ives, and S. J. Savory, "Differential carrier phase recovery for QPSK optical coherent systems with integrated tunable lasers," Opt. Express, vol. 21, no. 8, pp. 10166-10171, 2013. 